\title{
SFlex-WMS: a novel multi-expert system for flexible logistics and warehouse operation in the context of Industry 4.0
}

\author{
Sokchoo $\mathrm{Ng}^{1, *}$, Vin Cent Tai ${ }^{2}$, Yong Chai Tan², and Nor Faiza Abd Rahman ${ }^{2}$ \\ ${ }^{1}$ Faculty of Arts and Science, International University of Malaya-Wales, 50480 Kuala Lumpur, \\ Malaysia \\ ${ }^{2}$ Centre for Modelling and Simulation, SEGi University, 47810 Petaling Jaya, Selangor, Malaysia
}

\begin{abstract}
Production lines form the backbone of a manufacturing plant while the warehouse is the heart that pumps the supplies through logistics veins. However, logistics issues among manufacturing industries are well known for causing downstream production problems. Non-transparent warehouse operation and inevitable human error in logistics activities seriously jeopardise the entire downstream manufacturing processes. Existing warehouse management solutions require many sensors spanning the warehouse for tracking logistics activities which are cost-ineffective and inflexible. One aspect of intelligent WMS that has not been explored is the integration of computer vision modeling with Artificial Intelligence (AI) to create a more flexible, transparent, and autonomous warehouse management system (WMS). This study aims to devise a Smart and Flexible WMS (SFlex-WMS) to improve logistics operations in terms of operation costs, process time, and space utilisation. The highlight of the proposed framework is the two major work packages (WP) which focus on flexible, autonomous sensing mechanisms for inventory, logistics tracking, and space mapping, as well as reconstructing the warehouse environment model that reflects all physical changes in the warehouse. SFlex-WMS intends to realize real-time transparent monitoring of warehouse operations. By exploiting the outputs from both WPs, SFlex-WMS is expected to achieve more effective and flexible warehouse operations.
\end{abstract}

\section{Introduction}

The world is embarking on revolutionising the manufacturing industry once more. Known as Industry 4.0, its ultimate goal is to create smart factories through the use of cyber-physical systems, internet-of-things (IoT), cloud computing, and artificial intelligence (AI) to enable more flexible manufacturing and better adapt to market changes. On par with the paradigm shift in the manufacturing domain, Logistics 4.0 has been introduced to keep up with the industrial revolution (Strandhagen, Vallandingham, Fragapane \& Strandhagen, 2017). However, Small and Medium-sized Enterprises (SMEs) find it difficult to adopt the 4.0 concept due to high investment costs (Sommer, 2015; Schröder, 2016). Most SMEs are still

\footnotetext{
* Corresponding author: ashleyng@iumw.edu.my
} 
relying on labours to perform logistics activities in their production warehouse. Nontransparent warehouse operation and inevitable human error in the logistics activities severely jeopardise the entire manufacturing processes downstream. For example, misplaced items and containers can cause a significant amount of resource wastage in large automobile manufacturing plants (Zhou, Piramuthu, Chu \& Chu, 2017). Operational uncertainties from both demand and supply can also bring detrimental impact on logistics services that value high efficiency and low cost. As SMEs are the backbone of the economy (Karadag, 2016; Issa, Lucke \& Bauernhansl, 2017), there is an imminent need to provide them a more economical and effective means to solve the logistics problems.

SFlex-WMS seals the gaps between WMS and augmented reality to provide a real-time physical warehouse update by projecting the warehouse environment and setting in a flexible virtual model, thereby effectively eliminate human errors in the updating processes and enable transparent warehouse operations. The proposed smart "self-adjusting" warehouse aims to manage more operational randomness that serves as a characteristic to benefit warehouse management in SMEs.

\section{Related work}

Two classical decision hardships without regard to strategic, tactical, or operational level for a warehouse manager are warehouse management and inventory management problems (Fan \& Wang, 2017; Malaguti, Nannicini \& Thomopulos, 2018). For warehouse management issues, managers have to decide where to assign the goods inside the warehouse (Makaci, Reaidy, Evrard-Samuel, Botta-Genoulaz \& Monteiro, 2017). For inventory management, managers must decide which goods; and the quantity of each goods that need to be stored in the warehouse. So far, warehouse and inventory issues are handled in a pyramidal top-down approach where the flexibility of decisions decreases from top to bottom. As a scenario, when the design decision or size of the warehouse is fixed (top level), more restrictions are imposed to on the lower level decision, such as item replenish policy or area usage optimisation. The flexible warehousing concept (Zhou et al., 2017) was inspired by the Paris, Vélib' bicycles renting service in which the pick-up and drop-off of bicycles, or goods in the context of the warehouse, are at any (different) location in the network. To ensure the smooth operation, the system is required to always maintain a balanced ratio of the goods throughput and available space. Violation of such constraints such as empty or fully occupied space, will incur associated inconvenience in the logistics chain. For example, when there is not enough space for goods to arrive, the operator needs to arrange other nearby stations to store the goods. Thus, precise timing control of goods is crucial for such type of system.

Another warehouse management approach is the physical telephone directory system concept. This approach requires only a one-time initial setup cost to sort all names in alphabetical order and print the directory so that the cost of locating the goods in a later stage is minimised. Sequential database storage is implemented to realise such a system. Compared to flexible warehousing, the telephone directory or sequential database only does 'pick up' for once for every physical item instance from a given location. On the other hand, flexible warehousing imposes numerous 'pick up', which will take a toll on the logistics efficiency. More recent warehousing technology is Amazon's 'chaotic' warehouses which applies-apply bar-coded shelf location to store individual items regardless of their types (e.g., an electrical item can be stored next to a kitchen item) (Kumar, 2017). Other smart WMS is Lego's fully automated warehouses which have been operating without human intervention. What both fast fashion automated warehousing setting has in common is the demand location and quantity of goods are statistically consistent with the demands generated at the main inbound and outbound gates (Zhou et al., 2017). Zhou et al. (2017) found that such automated systems are relatively easy to manage due to their statistical certainty and limited number of goods 
variety. Recent improved flexible warehousing considers adopting RFID-tag to every item in the warehouse including products, tools and shelves. With the RFID-tag that stores information of the item, the location of each item can be traced in real-time with a fair degree of accuracy. Recent literature has affirmed that the future automated WMS is heading towards two major directions: 1) maximizing utilisation of warehouse capacity, and 2) warehouse item-handling operation efficiency (Alyahya, Wang \& Bennett, 2016; Joseph \& Joby, 2016; Tsao, Linh \& Lu, 2017; Yannick \& H. Ramzi, 2018).

As a summary, evidence has shown that the utilisation of RFID can expedite the automation of the warehouse storage and retrieval operations. Compared to the conventional WMS, Alyahya et al. (2016) claimed that the implementation of RFID systems has demonstrated a substantial improvement in warehouse data handling and space utilisation. With RFID technology, each item in the warehouse can be stored and dispatched in a storage rack at any random location where there is a space for incoming or outgoing goods (Alyahya, Wang \& Bennett, 2016). This plays an important part in reducing the operational randomness of a warehouse. Ideally, the RFID system can also interact with other control systems such as automated storage and retrieval to perform better warehouse monitoring in which items can easily be traced, sorted and inventoried in a real-time manner.

\section{SFlex-WMS}

SFlex-WMS (Fig. 1) is a multi-module framework for smart warehouse management in the context of Industry 4.0. This framework applies a data-driven mechanism that caters to the dynamic decision on location and local capacity demand in terms of warehouse space utilisation, inventory, and logistics handling. The main idea of SFlex-WMS design is to create a modular-based automated WMS by integrating edge computing, artificial intelligence, and state-of-art IoT technology into SME warehouse management. The working principle of the proposed modular-based framework is the whole framework is controlled by two work packages (WP) where each work package is formed by multiple modules (experts). Each individual module will take charge of a specific task. After completing the task, these modules (experts) will report back to their respective WP. Both of the WPs, playing the role of a warehouse manager, will integrate the data collected from experts, process, and issue the next instruction or action that needs to be taken to maintain the smooth running of the warehouse.

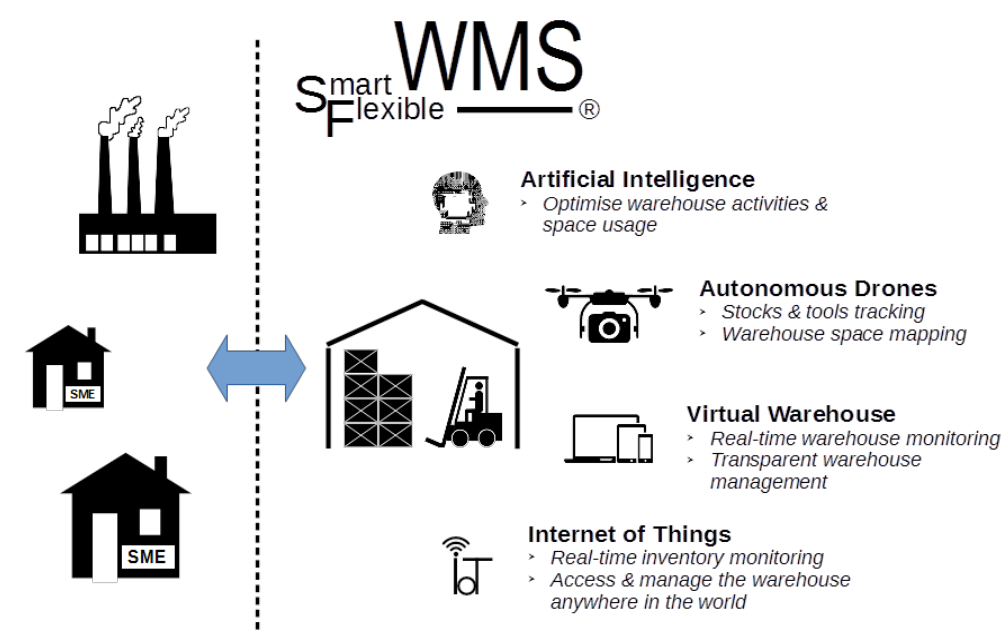

Fig. 1. Conceptual Design of SFlex-WMS 
This collaborative work breakdown structure of the software experts simulates the reallife human experts that focus on a specific task, and each outcome contributes to the success of the operational goal. The two major work packages (WP) are respectively in charge of 1) logistics tracking and space mapping and 2) warehouse environment modelling.

\subsection{WP-1: Multi-channel goods and storage identification}

Mathematically, the warehouse environment is perceived as a high dimensional multivariate problem space where it consists of multiple types of objects as unknown variables in a nonlinear problem space. WP-1 is known as Autonomous Drone Mapping \& Tracking. Autonomous in the current context means the WP is able to administer the navigation and space detection activities. It uses vision sensors, RFID, and drones for space mapping and goods tracking. WP-1 first enables a drone to navigate around the warehouse to capture the relevant data for the purpose of reconstructing the warehouse setting in the later part of modelling. During unmanned navigation, the drone is able to keep track of the path that it has passed through and at the same time avoid any obstacle along the path. In other words, the drone will not repeat the same pathway in order to save battery consumption. To prevent the done from being trapped in the same pathway all the time, evolutionary algorithms such as Genetic algorithms (GA) areis proposed to conduct the heuristic pathway searching in which the system will record the navigated path; meantime new pathways are consistently explored through the crossover and mutation mechanism. WP-1 architecture consists of four major modules known as expert (Fig. 2): (1) Object Identification Expert, (2) Space Mapping Expert, (3) RFID Tracking Expert and (4) Graphical User Interface (GUI).

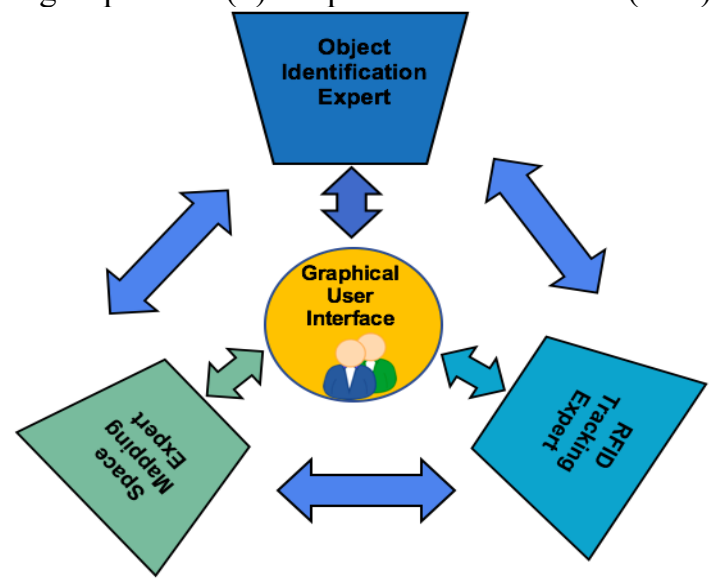

Fig. 2. WP-1 Autonomous Drone Mapping \& Tracking

The Object Identification Expert applies computer vision to recognise objects in an image or video sequence taken by the video camera which is attached to the drone. The working of the Object Identification Expert is based on the active vision approach, where the expert will actively select and analyse part of the visual scenes from the sensory apparatus. To meet the cost-effective challenge of the semi-structured industrial environment, multiple sensing channels (range sensor, RFID sensor and camera) are integrated to identify the randomly placed objects such as racks and goods as an individual object in the warehouse. The range sensor provides the distance information that complements the images captured by the camera. This is to ensure the expert produces more precise measurement and location information. The signal obtained from RFID sensor is used to itemize the goods in the warehouse. Based on the navigation path of the drone, the Space Mapping Expert starts to 
generate the map of the whole warehouse and storage space. A terrain map will be generated while the drone navigates through regions with large terrain variability and avoids the flat regions. The RFID Tracking Expert will be used to track, identify and locate goods attached with RFID tags in the warehouse. The data and information collected will be used as an input for WP-2 to visualise the warehouse environment.

\subsection{WP-2: Multi-model projection for the warehouse environment}

WP-2 develops a three-dimensional (3D) virtual environment to portray the actual warehouse layout and the goods and storage location setting. This modelling system consists of four major experts (Fig. 3): (1) Warehouse Layout Expert, (2) Goods \& Storage Layout Expert, (3) Dimension Projection Expert, and (4) Graphical User Interface. The Dimension Projection Expert first acquires the location and warehouse objects data from WP-1. The location data captured by WP-1 is basically the coordinates in point cloud (by the camera) and two-dimensional (2D) range view (by range sensor) format from the drone's spots of interest. These data do not represent the exact measurement of the warehouse environment. The main function of the Dimension Projection Expert is to recalculate the dimension of the warehouse, goods, and storage to their 1:1 ratio by referring to the data collected from WP1. Then, the exact measurement of the warehouse environment, goods, and storage will be sent to the Warehouse Layout Expert and Goods \& Storage Layout Expert, respectively, for further processing.

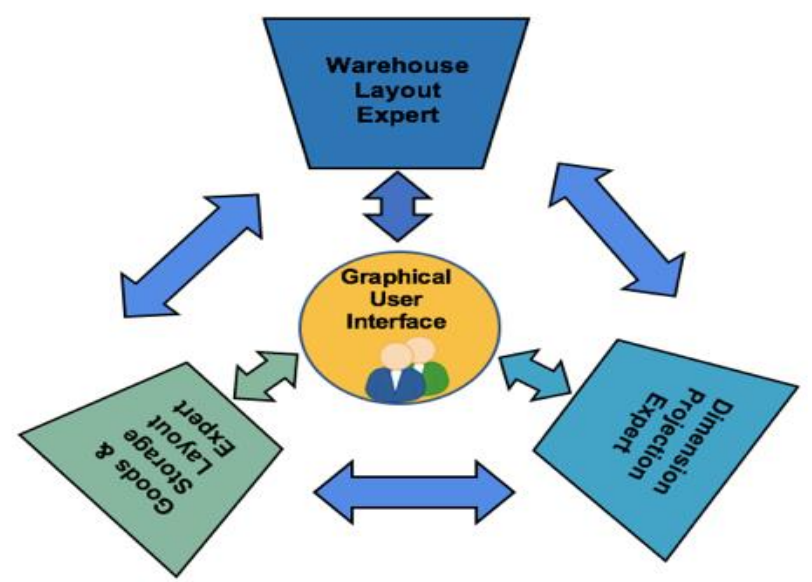

Fig. 3. WP-2 Multi-model Projection for Warehouse Environment

The Warehouse Layout Expert in charges of sketching the warehouse environment in its 3D architecture. A hybrid approach that combines view-based and graph-based methods will be applied to reconstruct the 3D model of the warehouse environment. The authors proposed that multiple views of the warehouse are captured from certain fixed viewpoints by a Kinect V2 camera. The visual similarity between the views of two models is regarded as the model similarity. By comparing via a kernel function, this model is then mapped into the topological graph structures from CAD drawing of the warehouse in order to form the $3 \mathrm{D}$ model. The Goods \& Storage Layout Expert renders the goods items and storage setting in 3D format by applying the same method. By combining the outcomes from both Warehouse Layout Expert and Goods \& Storage Layout Expert, the complete warehouse environment is projected. The user interacts with the system via a Graphical User Interface. 


\section{Simulation and testing}

The performance of the control algorithms developed in the previous sections can be tested and validated using Hardware-in-the-Loop (HiL) to ensure that they have similar real-time performance on the real proposed Smart WMS. Fig. 4 shows the flow chart of the dynamic stock storage \& retrieval and logistics management for WP at the real warehouse environment, in model space, and in HiL testing. The major highlight of WP is its loose coupling system design in which WP-1 and WP-2 are two major interrelated sub-systems, but they can be implemented and tested individually during the development. The rationale for this swarm robotics design is to ensure that WP can still function in the presence of partial failure. This is to mitigate the risk of any single failure point which could affect the normal operation of other parts of the system. The testing focuses on two warehousing productivity performance indicators (Karim, Abdul Rahman, Syed Johari Shah, 2018): inventory space utilisation and throughput. Taking one of the findings stated in Karim et al. (2009) as the benchmark, SFlex-WMS is expected to achieve at least $60 \%$ of inventory space utilisation at the same time minimising the mistake in the order picking process and bad inventory management.
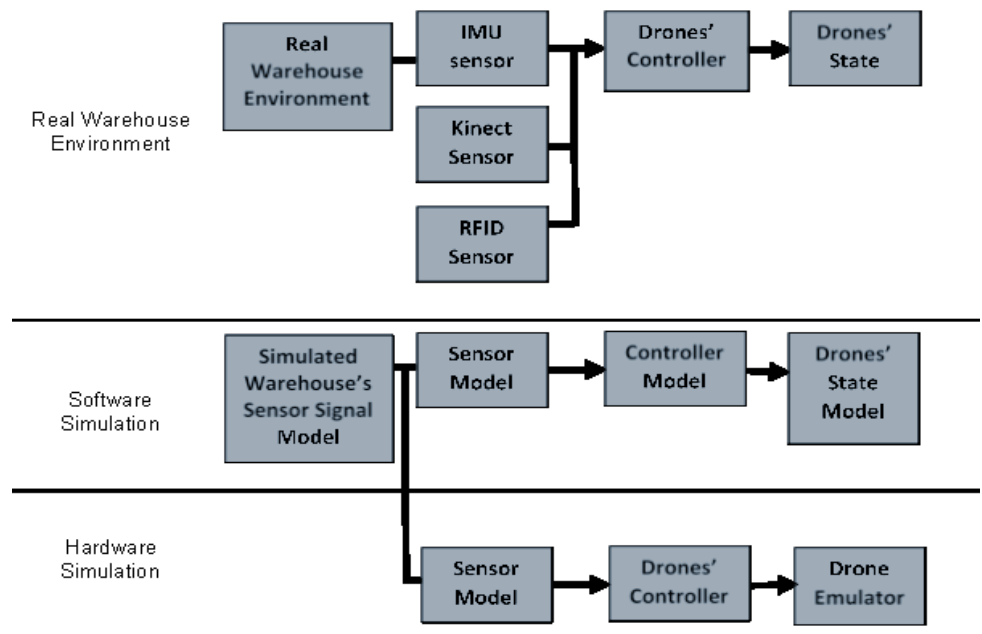

Fig. 4. Actual Warehouse, Software Simulation and Hardware Simulation Flowchart

\section{Conclusion}

The multi expert-based design of SFlex-WMS serves as a novel smart warehouse management system which closes the gap between the conventional warehouse operation and current technology. It demonstrates an integrated approach in modelling a warehouse setting and increasing information transparency in real-time logistics. A multi-model projection algorithm that combines 2D drawing with computer-aided graphic was proposed. The adoption of semantic-aware manual correction is a new approach to generate the Industry Foundation Classes-compliant 3D model of SME warehouse building. SFlex-WMS targets a more general type of warehouse with the following characteristics: 1) demands are created internally from regular job scheduling, and 2) the warehouse setting is subject to human intervention and other uncertainties. This type of warehouse can be seen in hardware manufacturing, repairing workshop or an overcrowded health care environment where the 
inventory buffer's capacity is low and sparse and has high uncertainty in demand. This new reusable and expandable WMS intends to increase warehouse flexibility, higher efficiency and reduced time to market of goods, and thus generate more business for sustainable socioeconomic development.

\section{ACKNOWLEDGEMENT}

This work is supported by FRGS under the project ref. FRGS/1/2018/TK03/SEGI/02/1.

\section{REFERENCES}

Alyahya, S., Wang, Q., \& Bennett, N. (2016). Application and integration of an RFID enabled warehousing management system - a feasibility study. Journal of Industrial Information Integration, 4, 15-25.

Fan, J., \& Wang, G. (2017). Joint optimization of dynamic lot and warehouse sizing problems. European Journal of Operational Research, 267(3), 849-854.

Issa, A., Lucke, D., \& Bauernhansl, T. (2017). Mobilizing SMEs towards Industry 4.0 enabled smart products. Procedia CIRP, 63, 670-674.

Joseph, S. A., \& Joby, N. J. (2016). Analyzing RFID Tags in a Distributed Environment. Procedia Technology, 24, 148 3-1490.

Karadag, H. (2016). The role of SMEs and entrepreneurship on economic growth in emerging economies within the post-crisis era: an analysis from Turkey. Journal of Small Business and Entrepreneurship Development, 4(1), 22 - 31.

Karim, N. H., Abdul Rahman, N. S. F., \& Syed Johari Shah, S. F. S. (2018). Empirical evidence on failure factors of warehouse productivity in Malaysian logistic service sector, The Asian Journal of Shipping and Logistics, 34(2), 151-160.

Kumar, Rajesh. (2017). Wealth creation by Amazon. In Rajesh Kumar (Ed.), Strategic Financial Management Casebook (pp. 413-440). Academic Press.

Makaci, M., Reaidy, P., Evrard-Samuel, K., Botta-Genoulaz, V., \& Monteiro, T. (2017). Pooled warehouse management: An empirical study. Computers \& Industrial Engineering, $112,526-536$.

Malaguti, E., Nannicini, G. \& Thomopulos, D. (2018). Optimizing allocation in a warehouse network. Electronic Notes in Discrete Mathematics, 64, 195-204.

Schröder, C. (2016). The challenges of Industry 4.0 for small and medium-sized enterprises. The Friedrich-Ebert-Stiftung. Bonn.

Sommer, L. (2015). Industrial revolution - Industry 4.0: Are German manufacturing SMEs the first victims of this revolution? Journal of Industrial Engineering and Management, 8(5), $1512-1532$. 
Strandhagen, J. O., Vallandingham, L. R., Fragapane, G., \& Strandhagen, J. W. (2017). Logistics 4.0 and emerging sustainable business models. Advances in Manufacturing, 5(4), $359-369$.

Tsao, Y. C., Linh, V. T., \& Lu, J. C. (2017). Closed-loop supply chain network designs considering RFID adoption. Computers \& Industrial Engineering, 113, 716-726.

Yannick, L. F. \& Ramzi, H. (2018). Order batching in an automated warehouse with several vertical lift modules: Optimization and experiments with real data. European Journal of Operational Research, 267(3), 958-976.

Zhou, W., Piramuthu, S., Chu, F., \& Chu, C. (2017). RFID-enabled flexible warehousing. Decision Support Systems, 98, 99-112. 